\title{
The role of GLP-1/GIP receptor agonists in Alzheimer's disease
}

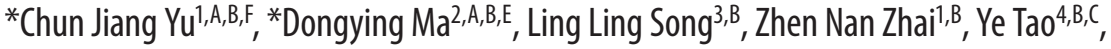 \\ Ying Zhang ${ }^{5, B, C}$, Ling Yu Cai ${ }^{6, B, C}$, Ya Hui Hou ${ }^{7, B, D}$, Hong Yuan Chen ${ }^{1, B, E}$, Li Wang ${ }^{8, A, C}$ \\ ${ }^{1}$ Department of Neurology, Second Affiliated Hospital of the Harbin Medical University, China \\ 2 Department of Neurosurgery, Second Affiliated Hospital of the Harbin Medical University, China \\ ${ }^{3}$ Department of Neurology, Second Hospital of Chaoyang City, China \\ ${ }^{4}$ Department of Neurology, First Hospital of Suihua City, China \\ ${ }^{5}$ Department of Neurology, People's Hospital of Hulunbuir, China \\ ${ }^{6}$ Department of Neurology, 117 hospital of PLA, Hangzhou, China \\ ${ }^{7}$ Department of Neurology, People's Hospital of the West Coast New District of QingDao, China \\ ${ }^{8}$ Department of Geriatrics, Second Affiliated Hospital of the Harbin Medical University, China \\ A - research concept and design; $\mathrm{B}$ - collection and/or assembly of data; $\mathrm{C}$ - data analysis and interpretation; \\ $\mathrm{D}$ - writing the article; $\mathrm{E}$ - critical revision of the article; $\mathrm{F}$ - final approval of the article
}

\section{Address for correspondence \\ LiWang \\ E-mail: liwang741@126.com}

\section{Funding sources}

The postdoctoral scientific research developmental fund of Heilongjiang Province (grant No. LBH-015100); postdoctoral scientific research developmental fund of Heilongjiang Province (grant No. LBH-Q18095); and National Natural Science Foundation of China (grant No. 61773134).

\section{Conflict of interest}

None declared

* These authors contributed equally to this work.

Received on May 9, 2019

Reviewed on May 30, 2019

Accepted on April 24, 2020

\section{Cite as}

Yu CJ, Ma D, Song LL, et al. The role of GLP-1/GIP receptor agonists in Alzheimer's disease. Adv Clin Exp Med. 2020;29(6):661-668. doi:10.17219/acem/121007

DOI

10.17219/acem/121007

\section{Copyright}

Copyright by Author(s)

This is an article distributed under the terms of the

Creative Commons Attribution 3.0 Unported (CC BY 3.0)

(https://creativecommons.org/licenses/by/3.0/)

\begin{abstract}
Background. New glucagon-like peptide-1 (GLP-1) analogues developed in recent years have a long halflife and offer further prospects for clinical application. At present, the neuroprotection of GLP-1 analogues in Alzheimer's disease (AD) has just begun to be explored.

Objectives. To investigate how glucagon-like peptide-1 (liraglutide) plays a protective role in AD by regulating tau activation and BACE1 expression.

Material and methods. Human neuroblastoma cell line SH-SY5Y cells were cultured in vitro and pretreated with different concentrations of liraglutide, and then treated with different concentrations of okadaic acid (OA) in order to observe the apoptosis of the SH-SY5Y cells. After liraglutide treatment, the apoptosis of neurons in AD rats was detected using flow cytometry, and tau activation and $\beta$-site APP cleaving enzyme 1 (BACE1) expression were detected using western blot.

Results. Different concentrations of OA were able to induce apoptosis of SH-SY5Y cells in a dose-dependent manner. Different concentrations of liraglutide were used to pretreat SH-SY5Y cells, which were able to protect the SH-SY5Y cells from apoptosis induced by OA. Okadaic acid significantly increased tau activation and BACE1 expression in the SH-SY5Y cells, which was blocked with liraglutide pretreatment. The results of a water maze experiment showed that liraglutide had significant protective effects on memory and cognitive ability in AD rats induced with $\mathrm{OA}$, inhibited apoptosis of neural cells in AD rats, and inhibited tau activation and BACE1 expression of neural cells in $A D$ rats induced with $O A$.
\end{abstract}

Conclusions. Liraglutide has a protective effect on $A D$ in vivo and in vitro, which may be mediated by preventing neuronal apoptosis and inhibiting the activation of tau and the expression of BACE1.

Key words: Alzheimer's disease, cognition, memory, nerve, glucagon-like peptide-1 


\section{Introduction}

Alzheimer's disease (AD) is a primary degenerative disease of the central nervous system which seriously affects quality of life. At present, there is no effective treatment method. ${ }^{1-3}$ It is becoming more and more urgent to find an effective treatment for AD. In recent years, glucagonlike peptide-1 (GLP-1) has been found to have neurotrophic and protective effects. ${ }^{4,5}$ The GLP-1 receptor activation can be used to treat type 2 diabetes mellitus. ${ }^{6}$ Because AD and type 2 diabetes mellitus share a common pathogenesis, the use of GLP-1 receptor activation in the treatment of AD has attracted the attention of scholars around the world. ${ }^{7}$ However, GLP-1 has a very short half-life in vivo, so it cannot be used as a drug to treat related diseases. However, the new GLP-1 analogues developed in recent years have a long half-life and offer more prospects for clinical application. At present, the neuroprotection of GLP-1 analogues on AD has just begun to be explored. There are few reports on the mechanism of GLP-1 analogues on AD protection.

Neuronal apoptosis is thought to be one of the major causes of neuronal loss in AD. Neurocyte apoptosis was first reported by $\mathrm{Su}$ et al. in 1994. It was found that there were fragments of DNA in the brain neurons of AD patients. Thus, the concept of neurocyte apoptosis was introduced into the neuropathology of AD. ${ }^{8}$ Tau proteins are microtubule-associated proteins with small molecular weight. The tau protein in the brain of AD patients is highly phosphorylated, ${ }^{9}$ and the phosphorylation of tau is regulated by protein kinase and protein phosphatase, the most important of which are protein phosphatase PP2A and protein kinase GSK-3 $\beta .{ }^{10,11}$ Protein kinase and protein phosphatase are in a dynamic balance in normal individuals, but the activity of PP2A in the brain of AD patients is significantly lower than that of normal adults. Tau in neurons is over-phosphorylated and can aggregate into a double helix and further forming neurofibrillary tangles. ${ }^{12} \beta$-site amyloid precursor protein (APP) cleaving enzyme 1 (BACE1) is a transmembrane aspartate protease that can cleave $\beta$-site APP to produce neurotoxic amyloid- $\beta(A \beta){ }^{13}$ The deposition of $A \beta$ in the brain is closely related to the development of AD. ${ }^{14,15}$

In this study, we explored the therapeutic effect of liraglutide on AD in vitro and in vivo by constructing an AD model, and we explored whether liraglutide played a role by regulating the process closely related to AD. This study found that liraglutide had a protective effect on $\mathrm{AD}$, which may be through the prevention of neuronal apoptosis, as well as inhibition of tau activation and BACE1 expression in nerve cells.

\section{Material and methods}

\section{Reagents}

SH-SY5Y cells were purchased from the Institute of Basic Medical Sciences, Chinese Academy of Medical
Sciences (Beijing, China). An annexin V/7AAD apoptosis staining kit was purchased from Biolegend company (San Diego, USA). Okadaic acid (OA) was purchased from Sigma-Aldrich (St. Louis, USA). Supersignal West Femto/ Pico HRP sensitive chemiluminescent substrate was purchased from Simmerfeld Technology Co., Ltd. (Beijing, China). Mouse anti-human pS396 tau antibody, mouse anti-human pS199/202 tau antibody and mouse anti-human BACE1 antibody were purchased from CST (New York, USA). C57BL/6 female rats were purchased from Beijing Weitonglihua Laboratory Animal Technology Co., Ltd. (Beijing, China; certificate No. CELAIC).

\section{SH-SY5Y cell culture}

The cells were cultured in Dulbecco's modified Eagle's medium (DMEM) (Thermo Fisher Scientific, Waltham, USA) containing $10 \%$ fetal bovine serum (FBS; Hyclone, Logan, USA). The medium was replaced every 2-3 days. The cells were pretreated with $0.1 \mu \mathrm{M}, 1 \mu \mathrm{M}$ and $10 \mu \mathrm{M}$ liraglutide for $72 \mathrm{~h}$, and then they were treated with $0 \mathrm{nM}$, $10 \mathrm{nM}$ and $20 \mathrm{nM}$ of OA for $48 \mathrm{~h}$.

\section{Flow cytometry}

The cells were collected at 1,000 g for $5 \mathrm{~min}$ and washed once with $1 \%$ phosphate-buffered saline (PBS). The cells were suspended with $100 \mu \mathrm{L}$ of binding buffer, and $5 \mu \mathrm{L}$ of annexin $V$ dye and $5 \mu \mathrm{L}$ of 7 -AAD dye were added. After incubating at room temperature for $15 \mathrm{~min}$, the cells detected using flow cytometry.

\section{Western blot}

The total protein of the cells was homogenized in cold radioimmunoprecipitation assay (RIPA) buffer (Beyotime, Shanghai, China). The total protein was centrifuged at $13,000 \times \mathrm{g}$ for $20 \mathrm{~min}$ at $4^{\circ} \mathrm{C}$. The supernatants were collected, and protein concentration was measured using a modified bicinchoninic acid (BCA) protein concentration assay kit (Beyotime) in accordance with the manufacturer's protocol. The extracted protein was separated using $8 \%$ SDS-PAGE (Bio-Rad, Hercules, USA) and 5\% concentrated gel (Bio-Rad) and transferred to nitrocellulose membrane (Simmerfelder Technology). The membrane was blocked with $5 \%$ bovine serum albumin (BSA) for $2 \mathrm{~h}$ and incubated with primary antibodies, including anti-pS396 tau, anti-pS199/202 tau and anti-BACE1, at $4{ }^{\circ} \mathrm{C}$ overnight. On the $2^{\text {nd }}$ day, the membrane was washed with $0.1 \%$ trisbuffered saline-Tween (TBST) 3 times, 5 min each time. Then, the membrane was incubated with the corresponding horseradish peroxidase (HRP)-conjugated secondary antibodies at room temperature for $1 \mathrm{~h}$. After being washed 3 times with $0.1 \%$ TBST, the signals were visualized using Supersignal West Femto HRP. Actin was used as an internal control. 


\section{Establishment of AD rat model}

The rats were randomly divided into a sham operation control group, a test group (OA group) and a liraglutide treatment group, with 10 rats in each group. The rats in the control group did not undergo any treatment. The rats in test and liraglutide treatment groups were injected with $0.5 \mu \mathrm{L}$ of OA dissolved in $10 \%$ dimethyl sulfoxide (DMSO) into the hippocampus. The concentration was $0.8 \mathrm{mmol} / \mathrm{L}$, and the injection was slowly performed at $0.1 \mu \mathrm{L} / \mathrm{min}$. The needle was kept in place for $10 \mathrm{~min}$, and then pulled out slowly. On the $16^{\text {th }}$ day after OA injection, the rats in the treatment group were subcutaneously injected with $300 \mu \mathrm{g} / \mathrm{kg}$ of liraglutide, and the rats in the control and test groups were injected with the same amount of saline solution for 30 days.

\section{Y-maze and Morris water maze}

The Y-maze consists of 3 equal-length arms $(50 \times 18 \times 35 \mathrm{~cm})$ with an angle of $120^{\circ}$ between each arm. Each arm has a movable partition in the center. The inner arm and bottom of the maze are painted black. Visual cues are placed on the walls of the 3 arms. After each test, the arm of the labyrinth was sprayed with alcohol to eliminate olfactory stimulation. When the spontaneous round examination was tested, the rats were placed at the end of one arm and allowed to explore for $8 \mathrm{~min}$ freely. The video camera system recorded the behavioral changes of the animals for $8 \mathrm{~min}$. The following parameters were recorded: 1) the total number of entries - the number of times that the animals entered the maze arm (with the 4 feet of a rat entering the arm counting as 1 time); 2) alternations - successively entering all 3 arms once; and 3 ) the number of maximum alternations - the total arm number was 2 . The percentage of the auto cycles is the total number of cycles/" $100 \%$.

The Morris water navigation test was performed according to the following procedure. The rats headed toward the wall of the pond, and randomly took 1 of the 4 starting positions - east, west, south, or north - into the water. The time the rats took to find an underwater platform(s) was recorded. In preceded training, if this time exceeded $60 \mathrm{~s}$, the rats were guided to the platform. Then, they were allowed to stay on the platform for $10 \mathrm{~s}$. The rats were removed and dried. When necessary, they were placed under an $150 \mathrm{~W}$ incandescent lamp for 5 min and then put back into the cage. Each rat was trained 4 times a day, the interval between 2 training sessions was 15-20 min, and the training lasted for 5 days. 3 . On the $2^{\text {nd }}$ day after the final training, the platform was removed and the $60 \mathrm{~s}$ training was initiated. The rats were placed into the water from the opposite side of the original platform quadrant. The time spent in the target quadrant (the quadrant where the platform was originally placed) was recorded, as well as the number of entries into the quadrant as a spatial memory index. After the $2^{\text {nd }}$ day of training, 4 days of counterpoint training began. The platform was placed in the quadrant of the original platform, and the method was the same as the acquired training. The rats were trained 4 times a day. The time and distance of swimming and the swimming speed were recorded each time.

\section{Statistical analysis}

SPSS v. 16.0 software (IBM Corp., Armonk, USA) was used to analyze the data, and the measurement data was described as means \pm standard deviation (SD). A t-test was used to compare the measurement data between the 2 groups. Comparisons of experimental groups were evaluated with one-way analysis of variance (ANOVA) followed by Bonferroni analysis. A p-value $\leq 0.05$ was considered statistically significant.

\section{Results}

\section{The protective effect of liraglutide on OA-induced apoptosis in SH-SY5Y cells}

The apoptotic rates of the SH-SY5Y cells treated with different concentrations of $\mathrm{OA}(0 \mathrm{mM}, 10 \mathrm{nM}$ and $20 \mathrm{nM})$ were $1.31 \pm 1.22,13.4 \pm 2.78$ and $21.47 \pm 4.21$, respectively ( $\mathrm{p}<0.05$; Fig. 1). According to these results, $10 \mathrm{nM}$ of $\mathrm{OA}$ is able to significantly induce apoptosis, so this concentration was used to induce apoptosis in SH-SY5Y cells in subsequent experiments. In addition, apoptosis of SH-SY5Y cells was detected after treatment with different concentrations of liraglutide $(0.1 \mu \mathrm{M}, 1 \mu \mathrm{M}$ and $10 \mu \mathrm{M})$. The apoptosis rates of SH-SY5Y cells were $12.93 \pm 3.12,7.34 \pm 2.98$ and $5.84 \pm 1.91$, respectively ( $<<0.05$; Fig. 1). Because the protective effects of $1 \mu \mathrm{M}$ and $10 \mathrm{M}$ of liraglutide on the apoptosis of SH-SY5Y cells were not statistically significantly different, $1 \mu \mathrm{M}$ was selected as the intervention dose in subsequent experiments.

\section{The effect of liraglutide pretreatment on OA-mediated activation of tau and BACE1 in SH-SY5Y cells}

As shown in Fig. 2A, the activation of tau phosphorylation in the SH-SY5Y cells was significantly upregulated by OA treatment, while the activation of tau phosphorylation induced by OA was significantly decreased by pretreatment with liraglutide $(\mathrm{p}<0.01)$. The expression level of BACE1 in the SH-SY5Y cells treated with OA was significantly upregulated by tau phosphorylation ( $<<0.01$; Fig. 2B). However, after pretreatment with liraglutide, the increased expression level of BACE1 induced by OA was significantly lower ( $<<0.01$; Fig. 2B). 
A

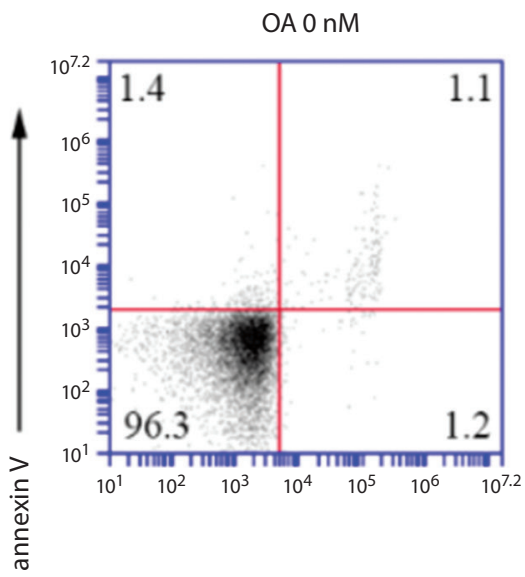

B

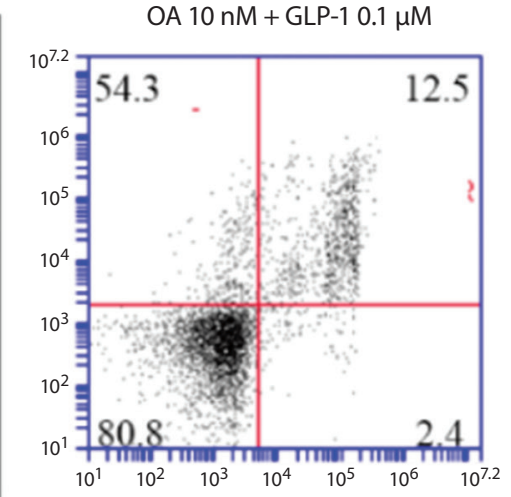

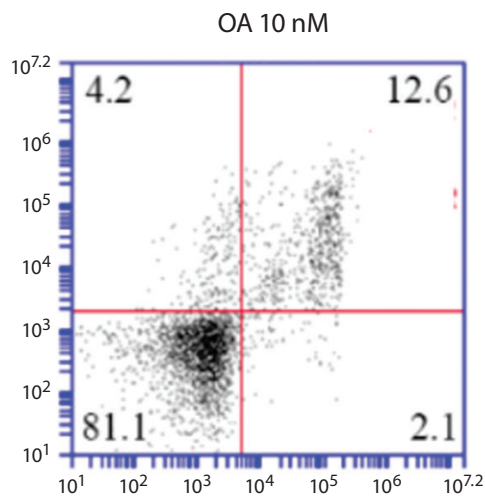

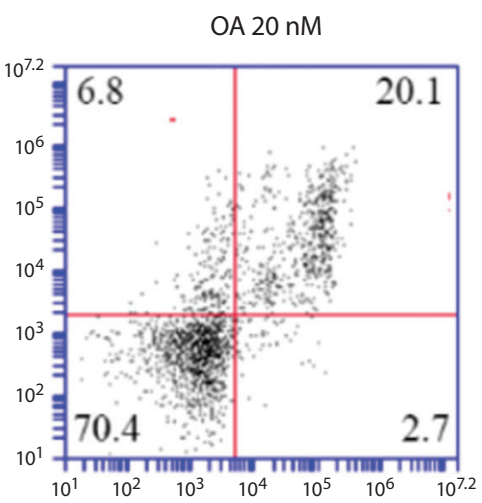

OA $10 n M+G L P-11 \mu M$

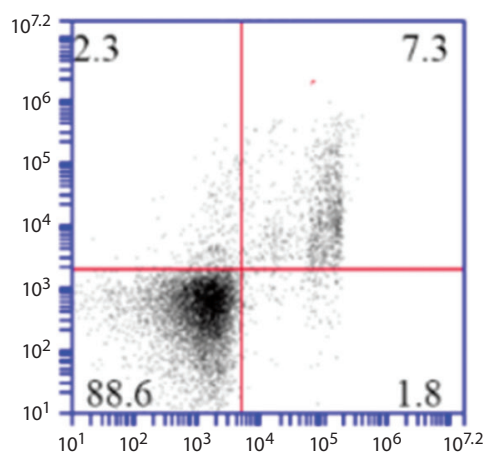

OA $10 \mathrm{nM}+\mathrm{GLP}-110 \mu \mathrm{M}$

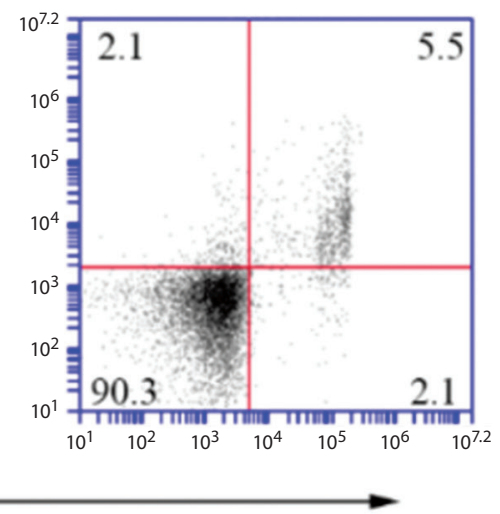

C

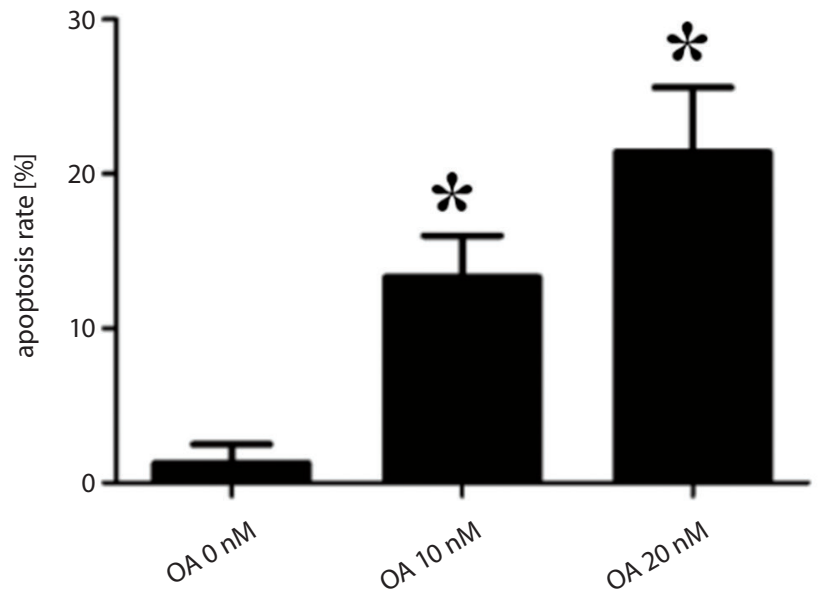

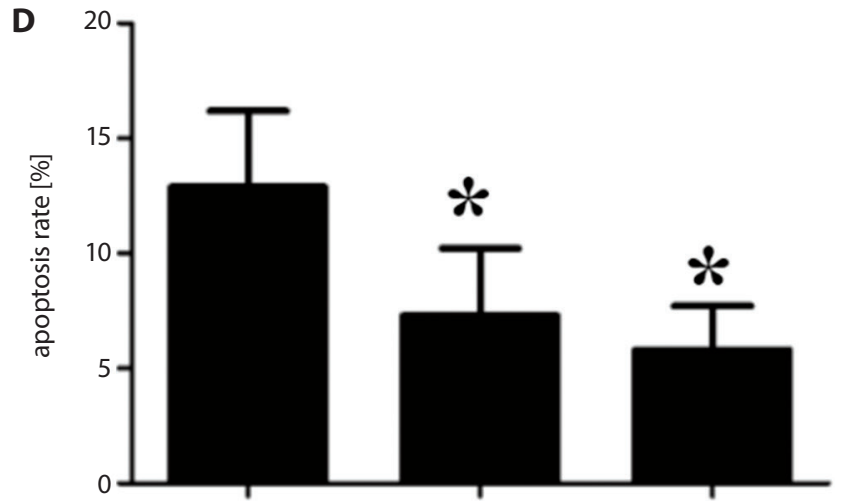

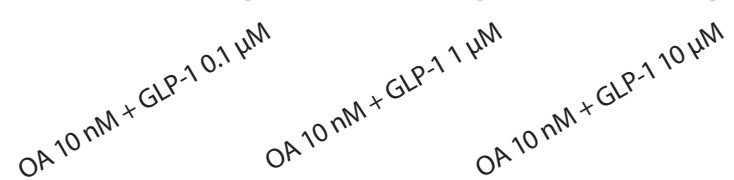

Fig. 1. Apoptosis of SH-SY5Y cells induced by OA and/or liraglutide was detected with apoptosis staining. A. The results of apoptosis staining induced with OA. B. The results of apoptosis staining induced with OA and liraglutide. C. The apoptosis rate of SH-SY5Y cells induced with OA. D. The apoptosis rate of SH-SY5Y cells induced with OA and liraglutide. Data are expressed as means \pm SD $(n=3)$

${ }^{*} \mathrm{p}<0.05$ vs the OA 0 nM group or the OA 10 nM+GLP-1 0.1 mM group.

\section{The learning and memory function of rats before and after treatment with liraglutide}

In order to detect the protective effect of liraglutide on OA-induced memory impairment, a Y-maze was used to test the ability of rats to recognize and remember a new environment. As shown in Fig. 3A, OA significantly affected the spontaneous behavior of rats compared with the control group, and this damage was significantly restored after treatment with liraglutide ( $\mathrm{p}<0.01$; Fig. 3A). However, there were no protective effects of liraglutide on OA-induced spatial recognition ( $p>0.01$; Fig. 3B). 
A

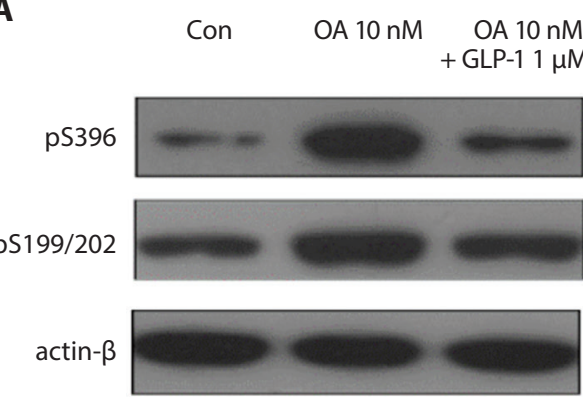

B

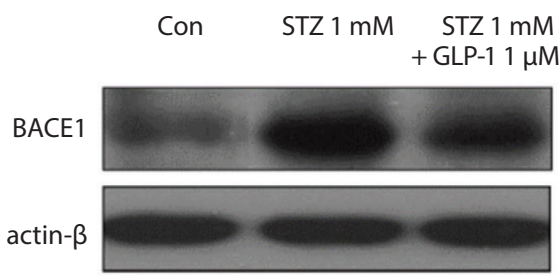

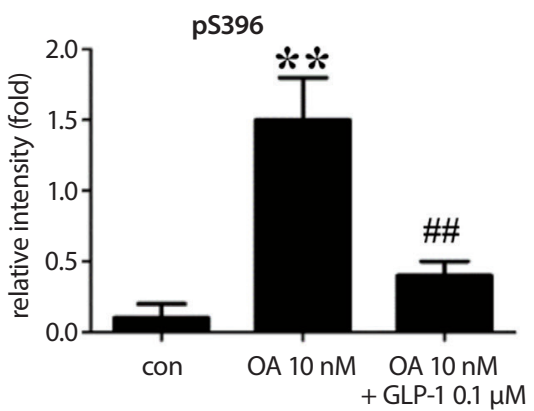
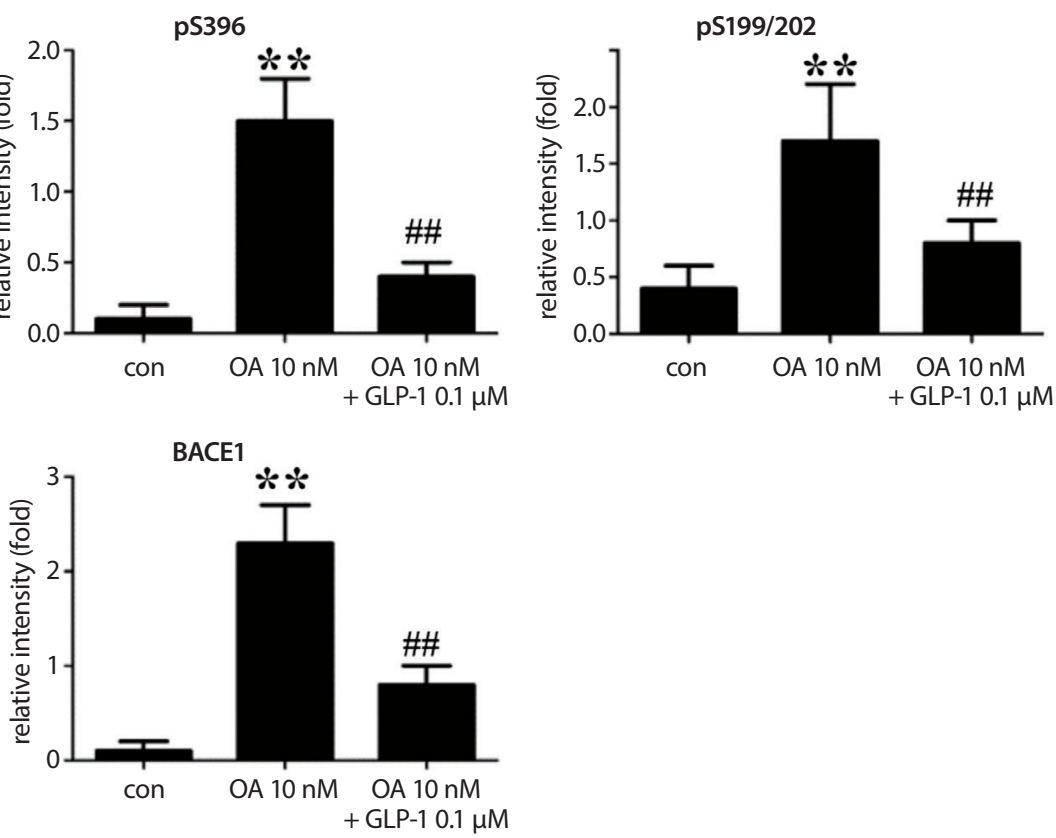

Fig. 2. The effects of liraglutide pretreatment on OA-mediated activation of tau and BACE1 in SH-SY5Y cells. A. Tau phosphorylation level. B. BACE1 expression level. Band intensities are normalized to actin- $\beta$ intensity and are expressed as relative values. Data are expressed as means \pm SD $(n=3)$

${ }^{*} \mathrm{p}<0.01$ vs the control group; ${ }^{* \#} \mathrm{p}<0.01$ vs the OA 10 nM group.

A

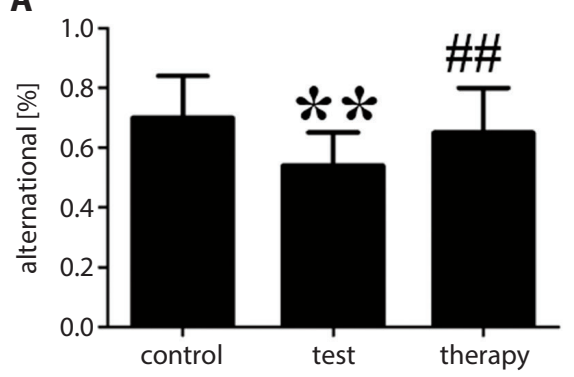

C

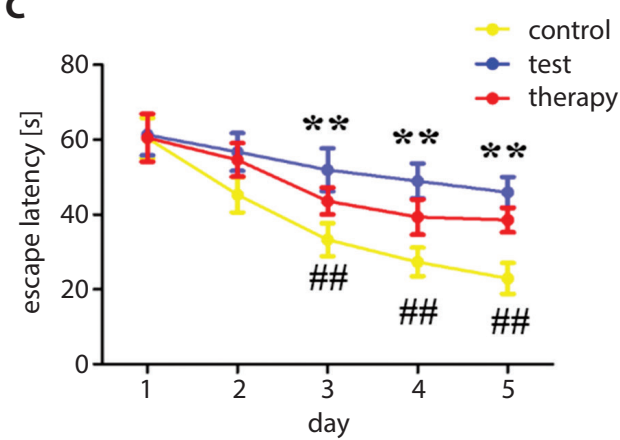

$\mathbf{E}$

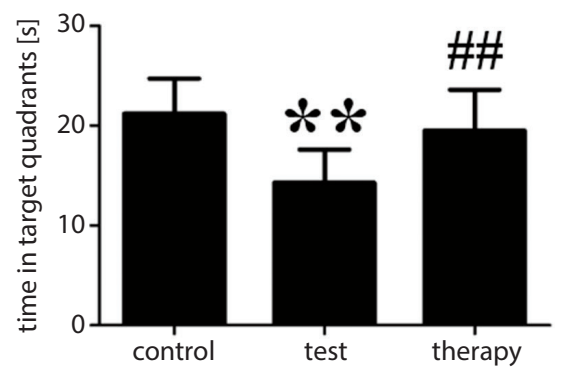

B

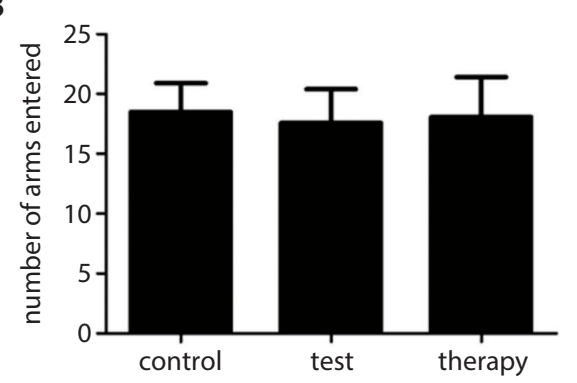

D

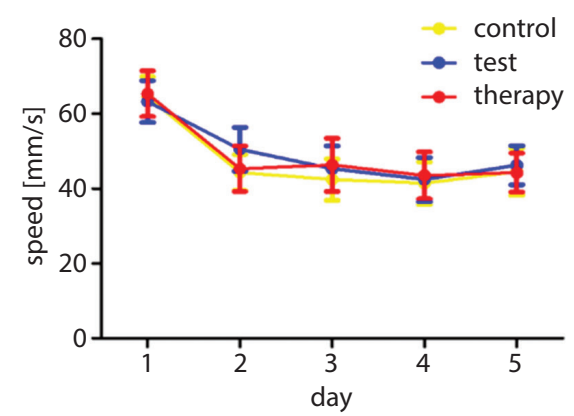

$\mathbf{F}$

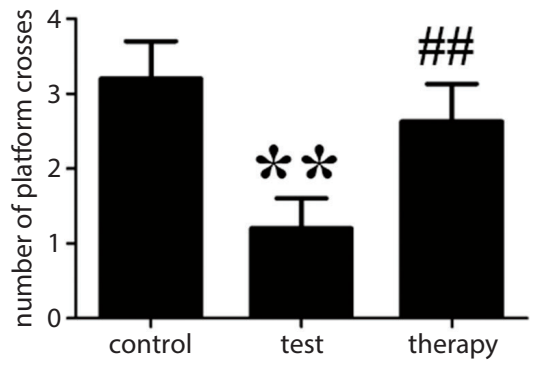

Fig. 3. Water maze test detection of learning and memory function in rats before and after treatment. A. Spontaneous Alternating Response Ability. B. Spatial Recognition Ability. C. Average Avoidance Latency of Hidden Platform. D. Swimming Speed. E. Residence Time in Target Quadrant. F. Platform Crossing Number. Data is expressed as means \pm SD

${ }^{* *} p<0.01$ vs the control group; $\# \mathrm{p}<0.01$ vs the test group (OA group). 

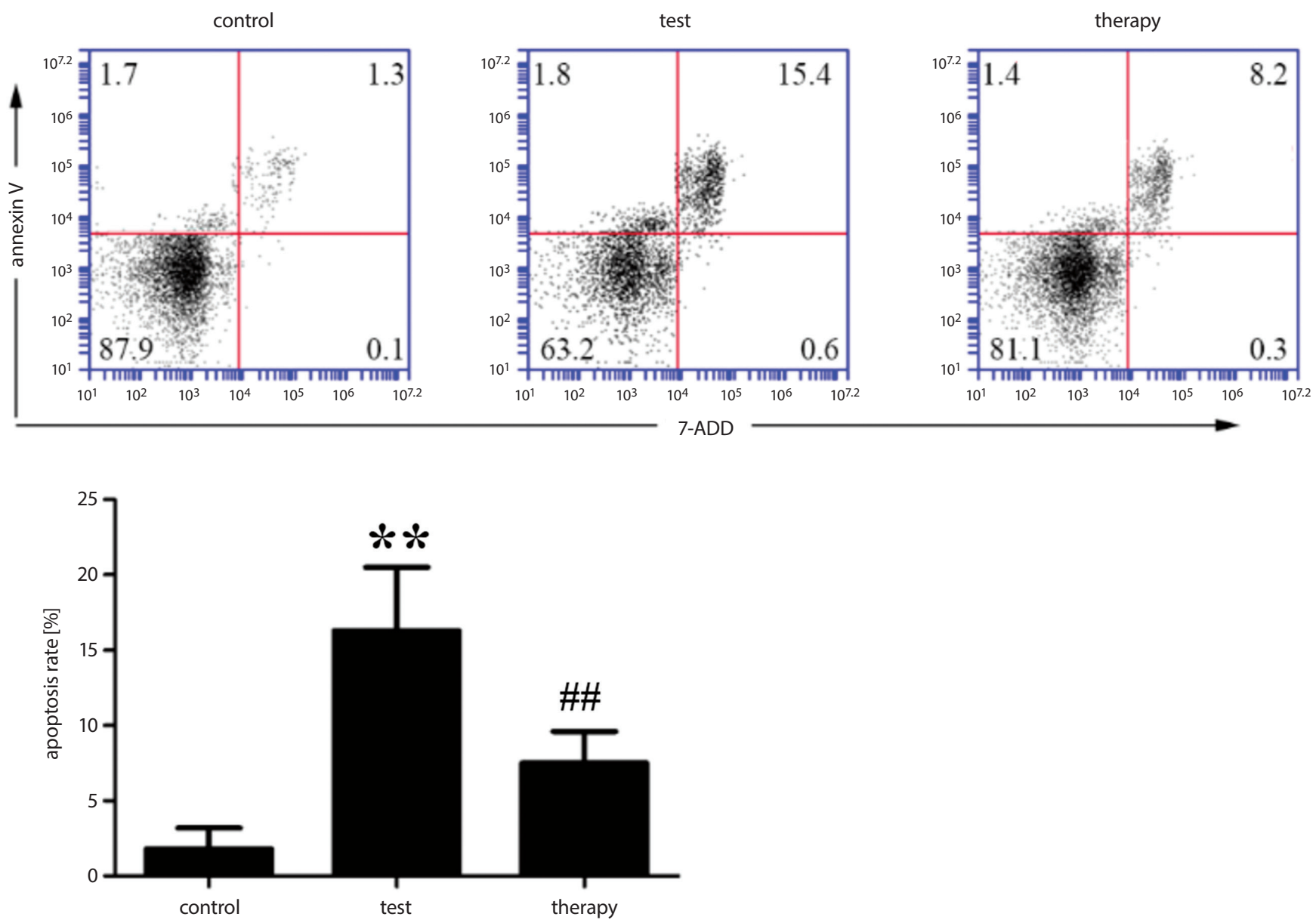

Fig. 4. The effects of liraglutide pretreatment on neuronal apoptosis in rats with AD. Data are expressed as means \pm SD

${ }^{* *} p<0.01$ vs the control group; $\#$ $p<0.01$ vs the test group (OA group).

A Morris water maze was used to detect the protective effect of liraglutide on OA-induced cognitive impairment. As shown in Fig. 3C, the average escape latency of the hidden platforms in the OA group was significantly higher, while it was significantly lowered when the treatment with liraglutide was employed ( $\mathrm{p}<0.01)$. However, there was no significant difference in swimming speed between the OA and the $\mathrm{OA}+$ liraglutide groups $(\mathrm{p}>0.01$; Fig. 3D). As shown in Fig. 3E and 3F, liraglutide shortened the residence time and decreased the number of platform crossings in the target quadrant of rats induced by OA $(\mathrm{p}<0.01)$. These results suggest that liraglutide has a significant protective effect on learning and memory impairment of AD rats induced with OA.

\section{Effect of liraglutide on neuronal apoptosis in $A D$ rats}

As shown in Fig. 4, the level of neuronal apoptosis in the test group was significantly higher than that of the control group $(1.84 \pm 1.41$ vs $16.36 \pm 2.14$; $\mathrm{p}<0.01)$. In addition, the level of neuronal apoptosis in the liraglutide treatment group was markedly lower when compared to the test group $(7.53 \pm 2.18$ vs $16.36 \pm 2.14 ; \mathrm{p}<0.01)$. These results indicate that liraglutide treatment can significantly reduce the level of neuronal apoptosis induced using OA in $\mathrm{AD}$ model rats.

\section{Effect of liraglutide on tau activation and BACE1 expression in neurons of AD rats}

As shown in Fig. 5A, the activation of tau phosphorylation induced by OA in the neurons of AD in a rat model was significantly higher, and when treated by liraglutide it was significantly inhibited $(\mathrm{p}<0.01)$. The change in BACE1 expression level was consistent with the activation of tau phosphorylation. The expression level of the BACE1 protein in the neurons of OA-induced AD rats was significantly higher, while this increase in BACE1 expression induced by OA was significantly inhibited after pretreatment with liraglutide $(\mathrm{p}<0.01$; Fig 5B).

\section{Discussion}

Alzheimer's disease, which accounts for $60-70 \%$ of dementia cases, is characterized by progressive loss of memory and cognitive abilities, as well as behavioral changes that 
A

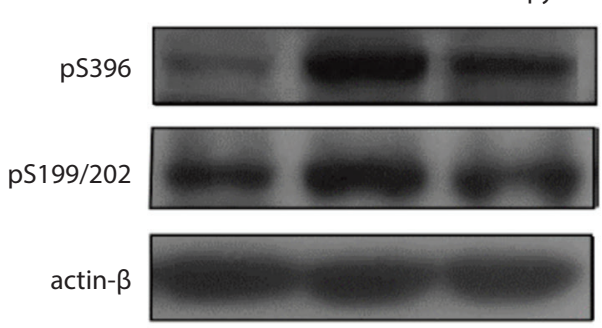

B

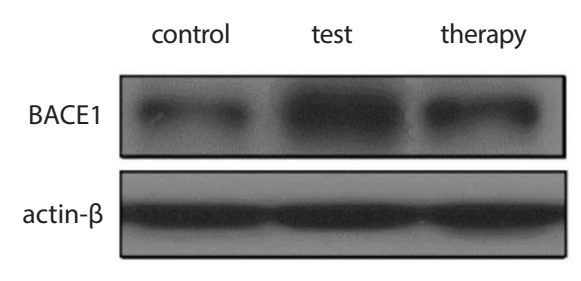

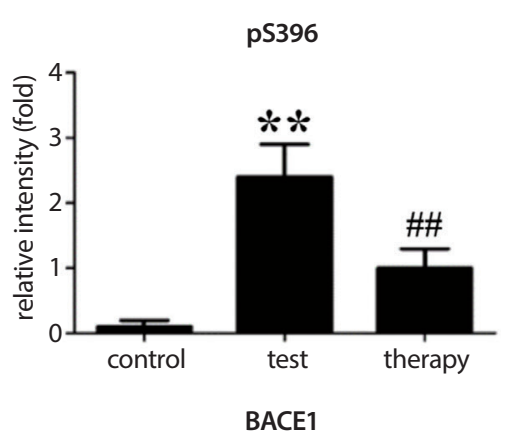

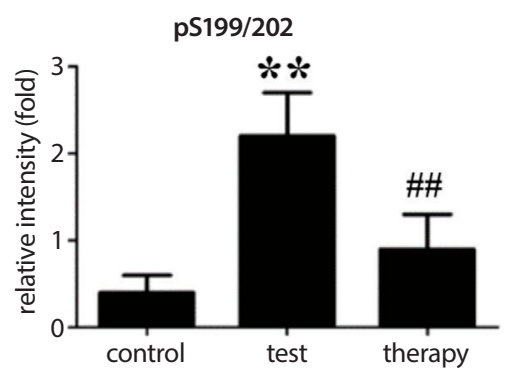

Fig. 5. The effects of liraglutide pretreatment on tau activation and BACE1 expression in the neural cells of rats with AD. A. Tau phosphorylation activation level. B. BACE1 protein expression level. Band intensities were normalized to actin- $\beta$ intensity and are expressed as relative values. Data is expressed as means $\pm S D(n=3)$

${ }^{* *} p<0.01$ vs the control group; $\#$ p $<0.01$ vs the test group (OA group).

ultimately lead to disability and dependence. ${ }^{16,17}$ At present, the treatment of AD can only alleviate the symptoms of patients, but cannot fundamentally changes the pathogenesis of AD. Therefore, it is very important to study the mechanism of AD. We carried out a preliminary study on the mechanism of liraglutide in the treatment of AD. The results showed that apoptosis of nerve cells was associated with $\mathrm{AD}$, which was mainly manifested in increased levels of apoptosis of the nerve cells in a rat model of $\mathrm{AD}$ in comparison with the control group. In vitro experiments showed that OA, which is able to induce apoptosis of SH-SY5Y cells, can also induce apoptosis in AD rats in a dose-dependent manner. At the same time, the experimental results confirmed that the occurrence of AD will be accompanied by an increase in tau phosphorylation activation levels and BACE1 protein expression levels. Furthermore, liraglutide had a certain therapeutic effect on $\mathrm{AD}$. In the water navigation task, the memory and cognitive function of rats treated with liraglutide were improved to a certain extent; this effect may come about through the prevention of neuronal apoptosis, the inhibition of the activation of tau and the expression of BACE1.

Okadaic acid, a protein phosphatase-2A (PP2A) inhibitor, has the effect of inducing tau protein hyperphosphorylation activation and inducing neuronal apoptosis. ${ }^{18,19}$ In vivo, OA stimulates the deposition of amyloid- $\beta(A \beta)$ and subsequently leads to neurodegeneration, synaptic loss and memory impairment, all of which are similar to those of AD. ${ }^{20}$ Okadaic acid has been shown to induce AD in rats. One of the key roles of OA in an AD model is that OA can induce tau protein hyperphosphorylation. ${ }^{21}$ This study confirmed that $\mathrm{OA}$ can induce tau protein hyperphosphorylation in SH-SY5Y cells in vitro, and in vivo experiments also confirmed that the phosphorylation and activation level of tau protein in rat neural cells was significantly higher in AD rats. We concluded that the phosphorylation activation of tau is closely related to the occurrence and development of AD. These results are consistent with the results of a previous study. ${ }^{22}$ We continued to observe the role of tau activation in the treatment of AD with liraglutide. It was found that liraglutide pretreatment significantly decreased the level of tau phosphorylation activation in SH-SY5Y cells induced by OA. As shown by in vivo experiments, liraglutide treatment can also inhibit the activation of tau phosphorylation, indicating that there was a certain correlation between the inhibition of tau activation and the therapeutic effect of liraglutide.

BACE1 was first discovered by Vassar in 1999 and has been proven to play an important role in the pathogenesis of $A D .{ }^{23}$ It can produce neurotoxic $A \beta$ by splitting the $\beta$-site APP. The deposition of $A \beta$ in the brain is closely related to the development of AD. ${ }^{14,15}$ Therefore, it can theoretically inhibit the production of $\mathrm{A} \beta$ by inhibiting $\mathrm{BACE} 1$ to treat $\mathrm{AD}$. In recent years, there have been many clinical studies on the use of BACE1 inhibitors in AD. Some of these studies have failed, while others are still observing efficacy. ${ }^{24}$ In addition to blocking the expression of BACE1 directly, other drugs can also inhibit the expression of BACE1 and have a certain therapeutic effect on AD. The results in vitro showed that liraglutide pretreatment could block the increase of BACE1 expression induced by OA. In vivo experiments have demonstrated that the level of BACE1 expression in the nerve cells of AD rats was lower in the treatment group. It is preliminarily suggested that another 
mechanism of liraglutide in treating AD may be through inhibiting the expression of BACE1 protein.

Both in vivo and in vitro studies have demonstrated that liraglutide has a protective effect on $\mathrm{AD}$, possibly by preventing neuronal apoptosis and inhibiting tau activation and BACE1 expression. The results provide a scientific basis for future research on AD therapy and its mechanism.

\section{ORCID iDs}

Chun Jiang Yu (1) https://orcid.org/0000-0003-4031-8657 Dongying Ma (10) https://orcid.org/0000-0003-3723-8170 Ling Ling Song (1) https://orcid.org/0000-0002-0555-1712 Zhen Nan Zhai (1) https://orcid.org/0000-0003-2585-8989 Ye Tao (i) https://orcid.org/0000-0002-8809-1626 Ying Zhang (1) https://orcid.org/0000-0002-9441-4591 Ling Yu Cai (i) https://orcid.org/0000-0003-4375-5801 Ya Hui Hou (1) https://orcid.org/0000-0002-6582-2529 Hong Yuan Chen (1) https://orcid.org/0000-0002-5941-3403 Li Wang (1) https://orcid.org/0000-0001-7248-7434

\section{References}

1. Auti ST, Kulkarni YA. A systematic review on the role of natural products in modulating the pathways in Alzheimer's disease. Int J Vitam Nutr Res. 2017;87(1-2):99-116.

2. Alasmari F, Ashby CR Jr, Hall FS, Sari Y, Tiwari AK. Modulation of the ATP-binding cassette $\mathrm{B} 1$ transporter by neuro-inflammatory cytokines: Role in the pathogenesis of Alzheimer's disease. Front Pharmacol. 2018;9:658.

3. Voss T, Li J, Cummings J, et al. Randomized, controlled, proof-of-concept trial of MK-7622 in Alzheimer's disease. Alzheimers Dement (N Y). 2018;4:173-181.

4. Knezovic A, Osmanovic Barilar J, Babic A, et al. Glucagon-like peptide-1 mediates effects of oral galactose in streptozotocin-induced rat model of sporadic Alzheimer's disease. Neuropharmacology. 2018; 135:48-62.

5. Li L. The molecular mechanism of glucagon-like peptide-1 therapy in Alzheimer's disease, based on a mechanistic target of rapamycin pathway. CNS Drugs. 2017;31(7):535-549.

6. Weisnagel SJ. The role of glucagon-like peptide-1 receptor agonists in cardiovascular disease prevention in type 2 diabetes mellitus: Evidence from the most recent clinical trials. Ann Trans/Med. 2018;6(10):194.

7. Athauda $D$, Foltynie $T$. The glucagon-like peptide 1 (GLP) receptor as a therapeutic target in Parkinson's disease: Mechanisms of action. Drug Discov Today. 2016;21(5):802-818.

8. $\mathrm{Su} \mathrm{JH}$, Anderson AJ, Cummings BJ, Cotman CW. Immunohistochemical evidence for apoptosis in Alzheimer's disease. Neuroreport. 1994; 5(18):2529-2533.
9. Mandelkow EM, Mandelkow E. Biochemistry and cell biology of tau protein in neurofibrillary degeneration. Cold Spring Harb Perspect Med. 2012;2(7):a006247.

10. Watkins GR, Wang N, Mazalouskas MD, et al. Monoubiquitination promotes calpain cleavage of the protein phosphatase $2 \mathrm{~A}$ (PP2A) regulatory subunit a4, altering PP2A stability and microtubule-associated protein phosphorylation. J Biol Chem. 2012;287(29):24207-24215.

11. Lee J, Hong H, Im J, Byun H, Kim D. The formation of PHF-1 and SMI-31 positive dystrophic neurites in rat hippocampus following acute injection of okadaic acid. Neurosci Lett. 2000;282(1-2):49-52.

12. Goedert M, Jakes R, Qi Z, Wang JH, Cohen P. Protein phosphatase 2A is the major enzyme in brain that dephosphorylates tau protein phosphorylated by proline-directed protein kinases or cyclic AMP-dependent protein kinase. J Neurochem. 1995;65(6):2804-2807.

13. Coimbra JRM, Marques DFF, Baptista SJ, et al. Highlights in BACE1 inhibitors for Alzheimer's disease treatment. Front Chem. 2018;6:178.

14. Wang $P$, Zheng $X, G u o Q$, et al. Systemic delivery of BACE1 siRNA through neuron-targeted nanocomplexes for treatment of Alzheimer's disease. J Control Release. 2018;279:220-233.

15. Andrew RJ, Fernandez CG, Stanley M, et al. Lack of BACE1 S-palmitoylation reduces amyloid burden and mitigates memory deficits in transgenic mouse models of Alzheimer's disease. Proc Natl Acad SciU S A. 2017;114(45):E9665-E9674.

16. Masters CL, Bateman R, Blennow K, Rowe CC, Sperling RA, Cummings JL. Alzheimer's disease. Nat Rev Dis Primers. 2015;1:15056.

17. De-Paula VJ, Radanovic M, Diniz BS, Forlenza OV. Alzheimer's disease. Subcell Biochem. 2012;65:329-352.

18. Kim D, Su J, Cotman CW. Sequence of neurodegeneration and accumulation of phosphorylated tau in cultured neurons after okadaic acid treatment. Brain Res. 1999;839(2):253-262.

19. Yoon S, Choi J, Yoon J, Huh JW, Kim D. Okadaic acid induces JNK activation, bim overexpression and mitochondrial dysfunction in cultured rat cortical neurons. Neurosci Lett. 2006;394(3):190-195.

20. Cho MH, Kim DH, Choi JE, Chang EJ, Seung-Yongyoon. Increased phosphorylation of dynamin-related protein 1 and mitochondrial fission in okadaic acid-treated neurons. Brain Res. 2012;1454:100-110.

21. Foidl BM, Humpel C. Differential hyperphosphorylation of tauS199, -T231 and -S396 in organotypic brain slices of Alzheimer mice. A model to study early tau hyperphosphorylation using okadaic acid. Front Aging Neurosci. 2018;10:113.

22. Vergallo A, Bun RS, Toschi N, et al. Association of cerebrospinal fluid a-synuclein with total and phospho-tau181 protein concentrations and brain amyloid load in cognitively normal subjective memory complainers stratified by Alzheimer's disease biomarkers. Alzheimers Dement. 2018;14(12):1623-1631.

23. Vassar R, Bennett BD, Babu-Khan S, et al. Beta-secretase cleavage of Alzheimer's amyloid precursor protein by the transmembrane aspartic protease BACE. Science. 1999;286(5440):735-741.

24. Coimbra JRM, Marques DFF, Baptista SJ, et al. Highlights in BACE1 inhibitors for Alzheimer's disease treatment. Front Chem. 2018;6:178. 